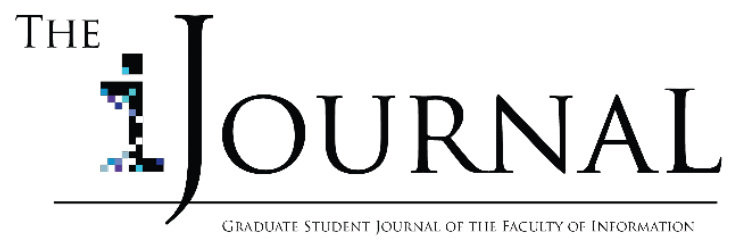

Lichty, David. (2020). The iJournal Vol 5, Issue 2, DOI:

10.33137/ijournal.v5i2.34415

\title{
Absorbing What is Useful:
}

Technology, Classification

Systems, and Intangible

Knowledge

David Lichty

\section{Abstract}

The following paper uses martial arts manuals to demonstrate the relationship between knowledge preservation and technology. It argues that technological constraints can influence the way information is organized, classified, and represented, thus affecting the way knowledge is translated into information. This paper explores how the technological limitations of a physical book cannot preserve the complexity of intangible knowledge in martial arts. The roles of hierarchy, transparency, standardization, and metadata are explored in the process of creating effective information-as-thing. Understanding the way technology affects the preservation of knowledge will better inform the preservation of intangible cultural knowledge in the cultural heritage field. Finally, a suggestion is made for an open-source website as an effective method for preserving intangible knowledge.

Keywords: Knowledge preservation, Martial arts, Classification, Intangible knowledge, Metadata

(C) 2020 Lichty, David. This is an Open Access article distributed under CC-BY.

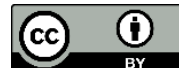


How does technology affect the way we preserve knowledge? This is a different question than asking how technology affects the way we preserve information. Information is physical; it exists in a tangible form. Knowledge, on the other hand, is not physical; it is ethereal in nature. Knowledge is just as much experience and understanding as it is interpreting the physical coding of information. As Michael Buckland (1991) puts it, knowledge can only be recorded as information, and information exists only as "information-as-thing" (p. 351). Whatever the effect technology has on preservation will have a direct bearing on the field of cultural heritage, as understanding how knowledge is translated into information through technology will better inform the practice of preserving intangible cultural knowledge.

In "A Thousand Times Do it: Historical European Martial Arts and the Cultural Record" (Lichty, 2020), I explore how intangible knowledge can be preserved through "information-asthing" (Buckland, 1989, p. 351). Through the analysis of historical European martial arts manuscripts, it is argued that the continued practice of intangible forms of knowledge (e.g. learning a language, martial art, or traditional dance), by a group of dedicated practitioners, is essential in improving the physical cultural record of intangible knowledge. However, I have come to realize that continued practice is not enough. What is also required is a classification system that accurately represents the intangible form of knowledge in question. This paper will suggest that the technologies used to preserve intangible forms of cultural knowledge affect how that knowledge is classified, organized, and represented. I intend to show this by analyzing how systems of classification can hinder the representation of intangible knowledge in the cultural record due to technological constraints. By exploring how modern martial arts techniques are organized in martial arts manuals, it is possible to see how the medium of the manual limits opportunities to overcome current assumptions and commitments inherent to modern martial arts. These biases create a problematic hierarchy of techniques that do not benefit the transfer of knowledge through a physical medium. Using the work of Geoffrey Bowker and Susan Leigh Star (1999), and Jens-Erik Mai (2010), I will demonstrate how the current classification systems used in manuals to represent and organize martial arts techniques do not achieve a desirable set of standards and level of transparency and therefore do not offer the best form of knowledge preservation. I will then suggest an alternative arrangement of martial arts techniques, one that will allow users to better preserve the practice of martial arts and other forms of intangible knowledge.

Let me begin by defining classification and its idealized practice so we can compare how martial arts manuals use classification methods to divide techniques into separate categories. Bowker and Star (1999) define classification as a set of categories into which things are separated and divided to create a form of knowledge production (p. 10). While this definition provides an important introduction to the idea of what classification is and does, they provide a more in-depth definition of what is normally seen as an ideal classification system. By deploying their critique of what they describe as an ideal, yet impossible, classification system, 
it is possible to identify how classification systems are ripe with bias and commitments. They outline the three qualities that are often associated with an ideal classification. The first is that all categories are consistent and follow a logical hierarchy. A hierarchy suggests only one route to success, that you can't deviate from the progression of skills as they are laid out. The second is that categories are mutually exclusive from one another, making the acquisition of one set of skills. The last quality is that a classification system provides a comprehensive method of categorizing that it sets out to classify. This suggests that deviation and seeking alternative information is unnecessary.

Modern martial arts manuals are an excellent example of classification in practice as outlined above. However, before I go into detail about how martial art manuals have inherent assumptions and commitments caused by their technological restraints, it is important to understand the nature of these manuals in general. Modern martial arts manuals are written by high-ranking and experienced practitioners of a particular martial art. The intention of creating modern martial arts manuals is to have an informative document on a specific martial art style, (e.g. karate, taekwondo, Brazilian jujitsu, boxing). This paper will focus specifically on martial art manuals for taekwondo and Brazilian jujitsu. These manuals are then divided into further categories based on the likeness of techniques. For example, in Starting in Taekwondo: Training for Competition \& Self-Defense (Fox \& Michaels, 1998) and The State of the Art: Taekwondo (Whang, Whang, \& Saltz, 1999), techniques are classified based on what they have predetermined as hand strikes, leg strikes, sparring techniques, and self-defence techniques. Manuals will then describe the execution of these techniques through text descriptions, pictures, and illustrations. Some martial arts manuals will divide techniques even further based on skill levels, as seen in Jiu-Jitsu University (Ribeiro \& Howell, 2008). Here, techniques are divided under belt ranks, with each belt representing a level of acquired skill. This idea of the progression of techniques also manifests through describing some techniques as basic/beginner or advanced.

While these manuals are successful in classifying techniques for the purpose of creating "information-as-thing" (Buckland, 1989), the limitations of the manual create problematic methods for classifying information. By examining how the classification systems in manuals attempt to realize the ideal qualities that Bowker and Star (1999) describe in their critique on classification systems, it becomes clear that the limitations of the medium influence how categories are defined. The result is a classification system for martial art techniques with practical limitations. The first flaw is that manuals are limited to the information the authors can provide. Often that manifests through the authors only having knowledge in one martial art or one methodology of training, creating a bias towards how techniques should be taught and practiced. For example, the roundhouse kick in many different martial arts is very similar if not the same, yet martial arts manuals are unable to refer to these similarities when it could be helpful for an individual seeking more relevant information because of the limited 
authorship in the production of the manual. While this distinction helps classification systems used in martial arts manuals to create mutually exclusive categories for their techniques, it cannot account for the possibility that different forms of intangible knowledge could be informative. The second assumption inherent in the classification of techniques is that there should be a logical hierarchy or genetic ordering of techniques while learning a martial art. JiuJitsu University (Ribeiro \& Howell, 2008) explains that belt promotion, which allows for the teaching of new techniques, is based on a prerequisite amount of knowledge for technical skill (p. 12). While demonstrating a progression or improvement of skill is important, it limits the amount of knowledge an individual can learn at one time. As Bowker and Star (1999) explain, " $[t]$ he decision to classify students by their standardized achievement and aptitude tests valorizes some kinds of knowledge skills and renders other kinds invisible" (p. 6). The last assumption inherent to martial arts manuals is that a physical document can provide complete coverage of the topics they try to classify. On the very cover of Taekwondo: The State of the Art (Whang, Whang, \& Saltz, 1999), it refers to itself as "[a] comprehensive instructional guide to the world's most popular martial art." This view assumes that no new techniques are going to be developed after the publishing of a martial art manual. This belief in a comprehensive classification system also does not account for regionalized variations, which are important for martial arts competitors to understand.

To further assess the assumptions inherent in the classification of martial arts techniques, it is important to evaluate how these classification systems are represented physically, and how these are limited by the technology of the physical manuals themselves. The issue with any enumerative system is that it becomes out of date the moment it is completed (Gartner, 2016, p. 78). Martial arts manuals cannot account for how martial arts techniques develop over time, thus limiting the interaction individuals can have with the manuals themselves. An effective strategy in martial arts competition five years ago would not be effective today, yet a martial arts manual cannot account for these changes. Emily Drabinsky (2013) states that classification structures should be in constant flux, and an effective approach must account for this issue (p. 108). The limited space in manuals causes one martial arts manual to omit or fail to acknowledge another, creating a "ghettoization" of martial arts techniques. Hope Olson and Rose Schlegl attribute "ghettoization" or a "problem of gathering and then isolating a topic rather than integrating it," to underlying assumptions within a classification system (Olson \& Schlegl, 2001, p. 67-68). It is easy to see how this occurs in martial arts manuals, which have to isolate techniques under the category of specific martial art styles and are unable to integrate techniques. Indeed, how what is made visible and what is made invisible is a common problem in the quality of knowledge produced by classification systems (Bowker \& Star, 1999, p. 5).

The result of having a classification system represented as a physical manual is that the assumptions and biases limit the use of metadata, thus restricting how effective the 
preservation of intangible knowledge can be. I want to propose that a more appropriate technology be used for martial arts techniques. Using a different type of technology to create information-as-thing would allow for transparency, user interaction, and using metadata through content-based retrieval. Using a different technology to create information-as-thing for martial arts techniques would better preserve the knowledge it attempts to represent. Rather than dividing the specific techniques under different martial arts categories such as style, the techniques would be compiled on a digital website with a format similar to Wiktenauer, a website that has compiled all medieval European martial techniques on a Wikipedia-style website ("Main Page," n.d.). Academics, practitioners, and experts are able to contribute related content and information. Each technique is listed independently because many of the techniques exist in many different styles, and each technique article can have hyperlinks connecting it to its variants, application, or parent martial art. I would suggest the same type of organization for the categorization of modern martial art techniques. With umbrella categories such as punching, kicking, grappling, submissions, throwing, and striking, the site would not limit users by isolating knowledge, nor would knowledge be invisible to the users.

Having these broad umbrella categories on a website would allow practitioners to make use of metadata when trying to learn an intangible form of knowledge. It would allow users of the website to avoid hierarchical restraints apparent in the current format of martial arts manuals, as you can "enter the web at any of these points and follow any route that leads from there" (Gartner, 2016, p. 82). A website also eliminates many of the problems created by the "ghettoization" of information: online data is interconnected and the lines between datasets are blurred and smudged (Gartner, 2016, p. 90). Each martial arts technique can be thought of as a piece of data and, as I have stated before, the isolation of techniques from one another limits how a practitioner can make use of the information. How this would be done is through hyperlinks connecting relevant information and content-based retrieval, as hyperlinks reflect "some judgment about two or more objects: they are the same, or alike, or functionally linked, or linked as part of an unfolding series" (Bowker \& Star, 1999, p. 7). Using a website also allows for the use of computer algorithms to locate related information to what the user would wish to find, allowing easier access to all the information at once.

Eliminating "ghettoization" by having interconnected datasets and search boxes allows more information to become visible to the user, thus solving the issue of invisibility that Bowker and Star (1999) mention. While making all information visible would be an impossible task, aiming to have a more transparent classification system would be an improvement, as transparent classifications are more trustworthy (Mai, 2010, p. 639). The importance of transparency does not merely end at allowing information to become visible. It is also necessary for users to be able to interact with a classification system and make decisions regarding the placement for information and the design of categories (Drabinsky, 2013, p. 
108). The arrangement that I suggest would allow for such a process. Just like Wiktenauer, practitioners would be able to have input and discussions with professionals about how information is being classified if they are the ones using that information to learn ("Main Page," n.d.). This level of interaction with the classification system would create a level of trust between the users and how the techniques are being classified, because the users are able to see the process used to categorize martial arts techniques.

My proposed arrangement of martial arts techniques on a website database tackles many of the issues created by the classification of techniques in martial arts manuals. It also achieves a higher set of standards according to the work of Bowker and Star (1999). The first standard is an agreed-upon set of rules regarding the production of objects (Bowker \& Star, 1999, p. 13). There is currently no such standard for the production of martial arts manuals. Each author can decide how they would like to design and classify information. A Wiktenauerstyle website would require all the information on the website to be formatted and presented in a consistent manner. The second is that standards should span beyond one community or practice (Bowker \& Star, 1999, p. 13). As I have argued, martial arts manuals by their very nature are written to exclude other martial arts styles. The arrangement I am suggesting would be inclusive of all martial arts, identifying the distinctive styles while also identifying their similarities. The interconnectedness that an online source will provide will address the physical limitation of these manuals. After all, standards help things work together over distance and heterogeneous metrics (Bowker \& Star, 1999, p. 13).

I want to emphasize why having a transparent and easily assessible classification system is an essential consideration in attending to how intangible knowledge is preserved in the cultural record. Through the exploration of historical martial arts manuscripts, we understand that the use of "information-as-thing" informs the creation of more reliable documents on the topic in the future (Lichty, 2020). This assumes that the classification system used to categorize intangible knowledge during this process would allow knowledge to be visible and easily interpreted. I want to propose that if the knowledge organized in "information-as-thing" limits the ability for a practitioner to make use of that knowledge, because some information is made invisible or information exists in isolation, qualitatively better "information-as-thing" will not be created in the future. Analyzing the underlying assumptions and biases of a classification system that attempts to organize intangible knowledge pertinent to the cultural record is crucial in ensuring that the cultural record becomes more informative over time. Scholars and practitioners need to be made aware of current best practices in recording and preserving information. Technologies used to preserve intangible cultural heritage need to be assessed as to how they might limit the categorization method. Without an iterative assessment of the application of these technologies, the intangible forms of knowledge they attempt to represent will be forgotten because of their lack of interpretability. This would be similar to how the techniques in early medieval martial arts manuscripts were forgotten for hundreds of 
years. Efforts to compensate for this loss are underway, but they are costly and fraught with difficulties.

"A Thousand Times Do it: Historical European Martial Arts and the Cultural Record" (Lichty, 2020) demonstrates why the continued practice of intangible knowledge is necessary for the development of an "information-as-thing" that is more usable and useful for those that interact with it. This paper takes that development one step further by demonstrating how classification systems can help or hinder the ways individuals interact with physical documents that categorize information representative of intangible knowledge. It is through the analysis of modern martial arts manuals that we can see how underlying assumptions and commitments can limit the visibility of relevant information and isolate other information. By understanding how these manuals are limited, I have suggested an alternative arrangement of organization that is dedicated to making information interconnected, accessible, and transparent. This is achieved by making use of metadata and content-based retrieval. To classify martial arts techniques effectively, practitioners need to be able to find relevant information, do away with problematic assumptions and biases, and contribute directly to an agile, online classification system. Alternatively, in the immortal words of Bruce Lee (2002, p.176), "absorb what is useful, reject what is useless and add what is essentially your own."

\section{References}

Bowker, G. C., \& Star, S. L. (1999). Sorting Things Out: Classification and its Consequences. The MIT Press.

Buckland, M. K. (1991). Information as thing. Journal of the American Society for Information Science (1986-1998), 42(5), 351. Retrieved from http://myaccess.library.utoronto.ca/login?url=https://search-proquestcom.myaccess.library.utoronto.ca/docview/216897238? accountid $=14771$

Drabinsky, E. (2013). Queering the Catalog: Queer Theory and the Politics of Correction. The Library Quarterly: Information, Community, Policy, 83(2), 94-111.

https://doi.org/10.1086/669547

Fox, J., \& Michaels, A. (1998). Starting in Taekwondo: Training for Competition \& SelfDefense. Sterling Publishing Company.

Gartner, R. (2016). Metadata: Shaping Knowledge from Antiquity to the Semantic Web. Springer. DOI: 10.1007/978-3-319-40893-4 [e-book]

Lee, B.,\& Little, J. (2002). Striking Thoughts: Bruce Lees Wisdom for Daily Living. Tuttle Publishing. 
LICHTY | ABSORBING WHAT IS USEFUL: TECHNOLOGY, CLASSIFICATION SYSTEMS, AND INTANGIBLE KNOWLEDGE

Lichty, D. (2020). A Thousand Times Do it: Historical European Martial Arts and the Cultural Record. The iJournal, 5(1), 28-34. https://doi.org/10.33137/ijournal.v5i1.33470

Mai, J. (2010). Classification in a Social World: Bias and Trust. Journal of Documentation, 66(5), 627-642. https://doi.org/10.1108/00220411011066763

Main Page. (n.d.). Retrieved from http://www.wiktenauer.com/

Olson, H. A., \& Schlegl, R. (2001). Standardization, Objectivity, and User Focus: A MetaAnalysis of Subject Access Critiques. Cataloging \& Classification Quarterly, 32(2), 618o. https://doi.org/10.1300/J104v32no2 06

Ribeiro, S., \& Howell, K. (2008). Jiu-Jitsu University. Victory Belt Publishing.

Whang, S. C., Whang, J. C., \& Saltz, B. (1999). Taekwondo: The State of the Art. Broadway Books. 